\title{
A new sufficient condition of optimality for the two-machine flowshop problem
}

\author{
C. Briand *, H.T. La, J. Erschler \\ LAAS-CNRS, 7 avenue du Colonel Roche, 31077 Toulouse, France
}

\begin{abstract}
This paper focuses on the characterization of a subset of optimal sequences for the famous two-machine flowshop problem. Based on the relative order of the job processing times, two particular interval structures are defined so that each job is associated with an interval. Then, using the Allen's algebra, the interval relationships are analysed and a sufficient optimality condition is established providing a characterisation of a large subset of optimal sequences. This set necessarily includes any Johnson's sequences together with numerous other optimal job sequences.
\end{abstract}

Key words: Scheduling, robustness, permutation flowshop, interval structures, pyramids.

\section{Introduction}

Scheduling methods able to provide a set of solutions as opposed to methods providing only one solution are said robust (or flexible) since they tolerate unforeseen disturbances without any schedule re-calculation [13]. According to this definition, several robust scheduling methods have been proposed in the literature $[17,18]$. Here under, the famous two machine flowshop problem is studied with the aim to characterize without enumeration a large set of optimal sequences. Classically, we denote $J$ the set of jobs to sequence on two machines $M_{1}$ and $M_{2}\left(p_{j 1}\right.$ and $p_{j 2}$ respectively indicate the processing times of each job $j \in J$ on $M_{1}$ and $M_{2}$ ). The selected criterion is the makespan $C_{\max }$ and only permutation sequences are considered (i.e. $F 2|p r m u| C_{\max }$ ) since they are dominant for $F m \| C_{\max }$, if $m \leq 3$ [12].

\footnotetext{
* Corresponding author.

Email addresses: briand@laas.fr (C. Briand), htla@laas.fr (H.T. La), erschler@laas.fr (J. Erschler).
}

Preprint submitted to European Journal of Operational Research 19 May 2004 
Several related papers already dealt with this problem providing a single or several optimal solutions. A well-known result was given by Johnson [19] who demonstrated that any job sequence which respects the rule $\min \left(p_{i 1}, p_{j 2}\right) \leq$ $\min \left(p_{j 1}, p_{i 2}\right) \Leftrightarrow i \prec j$ is optimal. Following this rule, a single optimal job sequence can be computed in $O(n \log n)$. The use of the algorithm proposed in [5] gives the list of all the sequences satisfying the Johnson's rule. Billaut and Lopez [6] also proposed an algorithm which enumerates, by job permutations in the optimal Johnson's sequences, the complete set of optimal sequences. A related approach based on the notion of maximal sequence was also proposed in [8]. In both cases, we notice that only small problems, with a dozen of jobs at most, can be solved due to a prohibitive time complexity of the algorithms. Indeed, the enumeration of the complete set of optimal sequences is a problem known to be NP-hard in the strong sense [16].

A $F 2|p r m u| C_{\max }$ problem possibly have a very large set of optimal solutions. The complete enumeration of this set has to be avoided since it is obviously time consuming. As a consequence, the approaches aiming at characterizing, rather than enumerating, a subset of optimal solutions should be preferred. This classically implies to handle sequential flexibilty by means of a partial order. A partial order $P$ is defined by a pair $P=\left(X, \preceq_{P}\right)$ where the binary relation $\preceq_{P}$ on $X \times X$ is reflexive, antisymetric and transitive [11]. A partial order $P=\left(X, \preceq_{P}\right)$ is a complete order (or a linear order) if for every pair $(u, v) \in X \times X$ either $u \preceq_{P} v$ or $v \preceq_{P} u$ holds. Moreover, given a pair of partial orders $Q=\left(X, \preceq_{Q}\right)$ and $P=\left(X, \preceq_{P}\right)$, on the same set $X$, we call $Q$ an extension of $P$ if $u \preceq_{P} v$ implies $u \preceq_{Q} v$ for all $u, v \in X$.

The notion of group sequence [22] gives an interesting partial order. It allows the characterization of a set of solution specifying, for each resource, an ordered sequence of group, provided that the order of the elements of a group is not imposed. The main advantage of such a partial order lies on the capability to perform a worst case analysis and to determine the quality of the set of solutions with respect to a regular criterion. Of course, the larger the set of solutions is and the worse its quality is. The notion of group sequence has been widely used in the field of shop scheduling and intends to determine a family of solutions to be used during the schedule real time execution. Switching from one solution to the other allows to face the potential disruptions without any peformance loss $[21,1,10,3,23,7,20]$.

In both [4] and [16], the authors suggest to reuse the notion of group sequence for the $F 2|p r m u| C_{\max }$ problem. The Baptiste's algorithm gives a group sequence characterizing a set of sub-optimal job sequences of which the worst execution time is bounded. On the basis of a given optimal job sequence, Esswein et al. also proposed a Greedy Forward Grouping algorithm, having the worst case complexity $O(n \log n)$, which determines an optimal group sequence of jobs while maximizing the flexibilty (i.e. the number of groups is 
minimized). In both previous works, the authors showed that the number of characterized optimal solutions can be very impressive.

The work presented in this paper is quite connected with the above approaches since we are also interested in characterizing a large set of optimal sequences for the $F 2 \mid$ prmu $\mid C_{\max }$ problem. Nevertheless, some new interesting features are provided. Firstly, a new partial order is defined which does not use the notion of group sequence. Moreover, our approach does not require any initial optimal sequence, since it can be directly stated that any sequence characterized with our partial order is optimal (the partial order is sufficient regarding the makespan criterion). Additionally, our partial order being defined on the basis of the relative order of the processing times $p_{i 1}$ and $p_{i 2}$, the characterized set of sequences remains optimal even if the job processing times increase, provided that the relative order is unchanged. Such a feature is of interest in a robust context where scheduling approaches have to be quite insensitive to data variations. Also interesting is the fact that Johnson's sequences are always included in the set of characterized sequences.

The paper is organised as follows. First we recall some basic notions, used for the definition of our partial order and related to the analysis of interval structures. Then we focus on two particular interval structures for the F2 $\mid$ prmu $\mid C_{\text {max }}$ problem and we progressively expound several sufficient conditions of optimality, starting from the analysis of particular interval structures up to the general case. Lastly, an example is given.

\section{Interval structure and basic concepts}

An interval structure can be defined by a couple $<I, C>$ with $I=\left\{i_{1}, \ldots, i_{n}\right\}$ a set of intervals and $C$ a set of constraints over $I \times I$. Each interval $i_{j}$ is defined by its lower and upper bounds $x_{j}$ and $y_{j}$. Any constraint between two intervals $i_{j}$ and $i_{k}$ can be expressed either by specifying a total order relation among the lower and upper bounds of the intervals or by directly using the relations of the algebra proposed by Allen [2] (see figure 1).

For instance, let us consider the interval structure with $I=\{A, B, C, D, E, F, G\}$ of the figure 2. We assume that $C$ is defined by a total order such that $\left(x_{A}=x_{B}=x_{G}\right)<\left(x_{C}=x_{D}\right)<y_{C}<\left(y_{B}=x_{F}\right)<x_{E}<y_{A}<\left(y_{D}=\right.$ $\left.\left.y_{E}\right)<y_{F}=y_{G}\right)$. The set of equivalent Allen's relations is represented on the table of the figure 3 . Such a table can be computed with a time complexity $O(n(n-1) / 2), n$ being the number of intervals.

Top and base [15] are two interesting notions related to the concept of interval structure. 


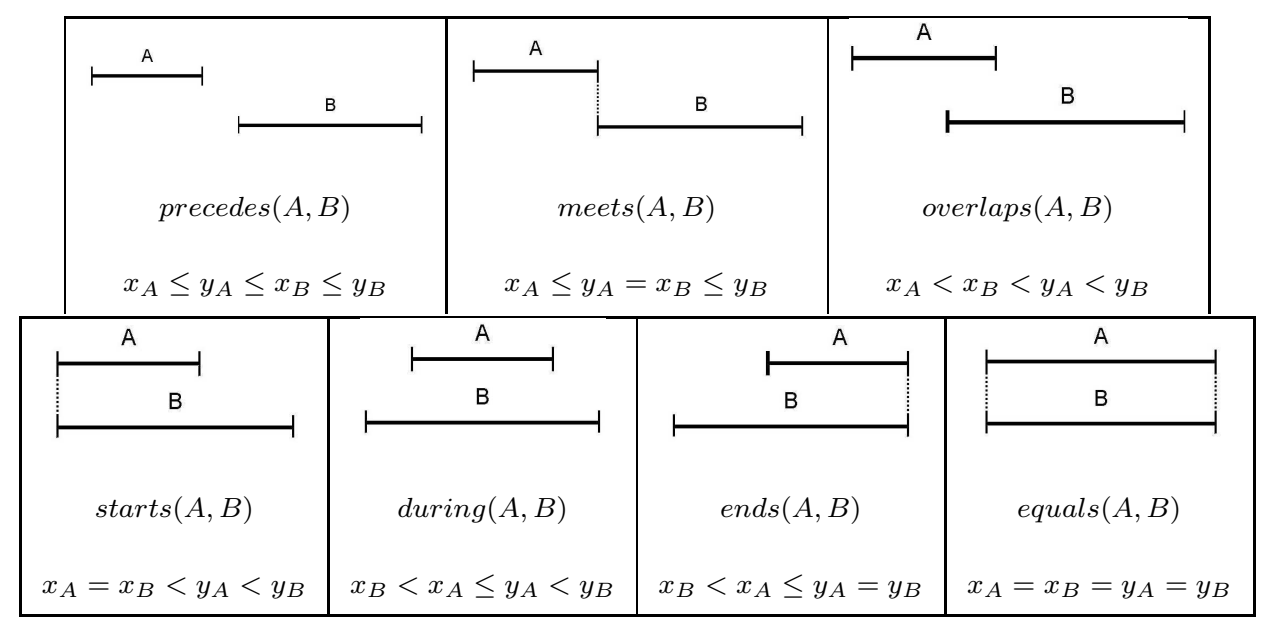

Fig. 1. Allen's relations

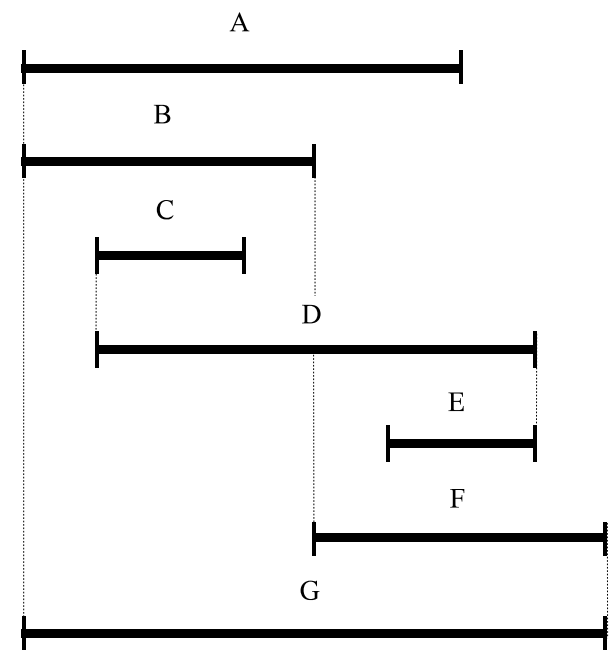

Fig. 2. An interval structure example

\begin{tabular}{l|lllllll} 
& $A$ & $B$ & $C$ & $D$ & $E$ & $F$ & $G$ \\
\hline$A$ & equals $(A, A)$ & starts $(B, A)$ & during $(C, A)$ & overlaps $(A, D)$ & overlaps $(A, E)$ & overlaps $(A, F)$ & starts $(A, G)$ \\
$B$ & $\operatorname{starts}(B, A)$ & equals $(B, B)$ & during $(C, B)$ & overlaps $(B, D)$ & precedes $(B, E)$ & meets $(B, F)$ & starts $(B, G)$ \\
$C$ & during $(C, A)$ & during $(C, B)$ & equals $(C, C)$ & starts $(C, D)$ & precedes $(C, E)$ & precedes $(C, F)$ & during $(C, G)$ \\
$D$ & overlaps $(A, D)$ & overlaps $(B, D)$ & starts $(C, D)$ & equals $(D, D)$ & ends $(E, D)$ & overlaps $(D, F)$ & during $(D, G)$ \\
$E$ & overlaps $(A, E)$ & precedes $(B, E)$ & $\operatorname{precedes}(C, E)$ & ends $(E, D)$ & equals $(E, E)$ & during $(E, F)$ & during $(E, G)$ \\
$F$ & overlaps $(A, F)$ & meets $(B, F)$ & precedes $(C, F)$ & overlaps $(D, F)$ & during $(E, F)$ & equals $(F, F)$ & ends $(F, G)$ \\
$G$ & $\operatorname{starts}(A, G)$ & starts $(B, G)$ & during $(C, G)$ & during $(D, G)$ & during $(E, G)$ & ends $(F, G)$ & equals $(G, G)$
\end{tabular}

Fig. 3. Allen's relation for the intervals $\{A, B, C, D, E, F, G\}$

Definition 1. A top of an interval structure $\langle I, C\rangle$ is an interval $t \in I$ such that $\forall i \in I$ the Allen's relation during $(i, t)$ never holds.

Definition 2. A base of an interval structure $\langle I, C\rangle$ is an interval $b \in I$ such that $\forall i \in I$ the Allen's relation during $(b, i)$ never holds.

These notions of top and base can be respectively used to define the notions of t-pyramid and b-pyramid. 
Definition 3. Given a top $t_{\alpha}$, a t-pyramid $P_{\alpha}$ related to $t_{\alpha}$ is the set of intervals $i \in I$ such that during $\left(t_{\alpha}, i\right)$.

Definition 4. Given a base $b_{\alpha}$, a b-pyramid $P_{\alpha}$ related to $b_{\alpha}$ is the set of intervals such that during $\left(i, b_{\alpha}\right)$.

For illustration, let us consider the interval structure of the figure 2. It has three tops $\{C, D, E\}$ and four bases $\{A, B, F, G\}$. The involved t-pyramids are $P_{C}=\{B, A, G\}, P_{D}=\{G\}$ and $P_{E}=\{F, G\}$, and the b-pyramids are $P_{A}=\{C\}, P_{B}=\{C\}, P_{F}=\{E\}$ and $P_{G}=\{C, D, E\}$.

The concept of t-pyramid has been already used in the scheduling literature in order to determine a dominant partial order [14,9] for the single machine problem $\left(1\left|r_{i}, d_{i}\right| L_{\max }\right)$. In the following, the focus is put on the b-pyramid concept which gives a sufficient condition of optimality for the $F 2 \mid$ prmu $\mid C_{\max }$ problem.

\section{Two interval structures for $F 2|p r m u| C_{\max }$}

Two particular interval structures $I_{1}$ and $I_{2}$ are defined:

- $I_{1}$ is the interval structure associated with the jobs $j \in J$ such that $p_{j 1} \leq p_{j 2}$;

- $I_{2}$ is the interval structure associated with the jobs $j \in J$ such that $p_{j 2} \leq p_{j 1}$.

An interval $i_{j}=\left[p_{j 1}, p_{j 2}\right]$ is associated to each job $j \in I_{1}$ and similarly, an interval $i_{j}=\left[p_{j 2}, p_{j 1}\right]$ is associated to each job $j \in I_{2}$. Therefore, a job $j$ such that $p_{j 1}=p_{j 2}$ belongs to both interval structures $I_{1}$ and $I_{2}$ and its corresponding interval is a point. Moreover, one can see that the interval structures $I_{1}$ and $I_{2}$ do not change as long as the relative order of the processing times $p_{j 1}$ and $p_{j 2}$ remains unchanged.

In the following, we focus on the bases of $I_{1}$ and $I_{2}$. We assume that the $n_{1}$ bases of $I_{1}$ are indexed according to the ascending order of their processing times on the first machine (in an arbitrary order in case of equality). Similarly, the $n_{2}$ bases of $I_{2}$ are indexed, starting from the index $n_{1}+1$, according to the descending order of their processing times on the second machine (in an arbitrary order in case of equality).

For the problem instance of the figure 4, the interval structures $I_{1}$ and $I_{2}$ are represented on the figure 5 . First, we notice that $I_{1}$ contains three bases, $b_{1}=1, b_{2}=2$ and $b_{3}=3$, which involve three b-pyramids: $P_{1}=\{5,6\}$, $P_{2}=\{6,7\}$ and $P_{3}=\{8\}$. The interval structure $I_{2}$ only contains a single base, $b_{4}=4$, which involves the b-pyramid $P_{4}=\{7,9\}$. 


\begin{tabular}{l|c|c|c|c|c|c|c|c|c} 
jobs $j$ & 1 & 2 & 3 & 4 & 5 & 6 & 7 & 8 & 9 \\
\hline$p_{j 1}$ & 1 & 3 & 8 & 8 & 2 & 4 & 7 & 9 & 5 \\
\hline$p_{j 2}$ & 6 & 8 & 12 & 2 & 4 & 5 & 7 & 11 & 3
\end{tabular}

Fig. 4. A $F 2 \mid$ prmu $\mid C_{\max }$ problem instance
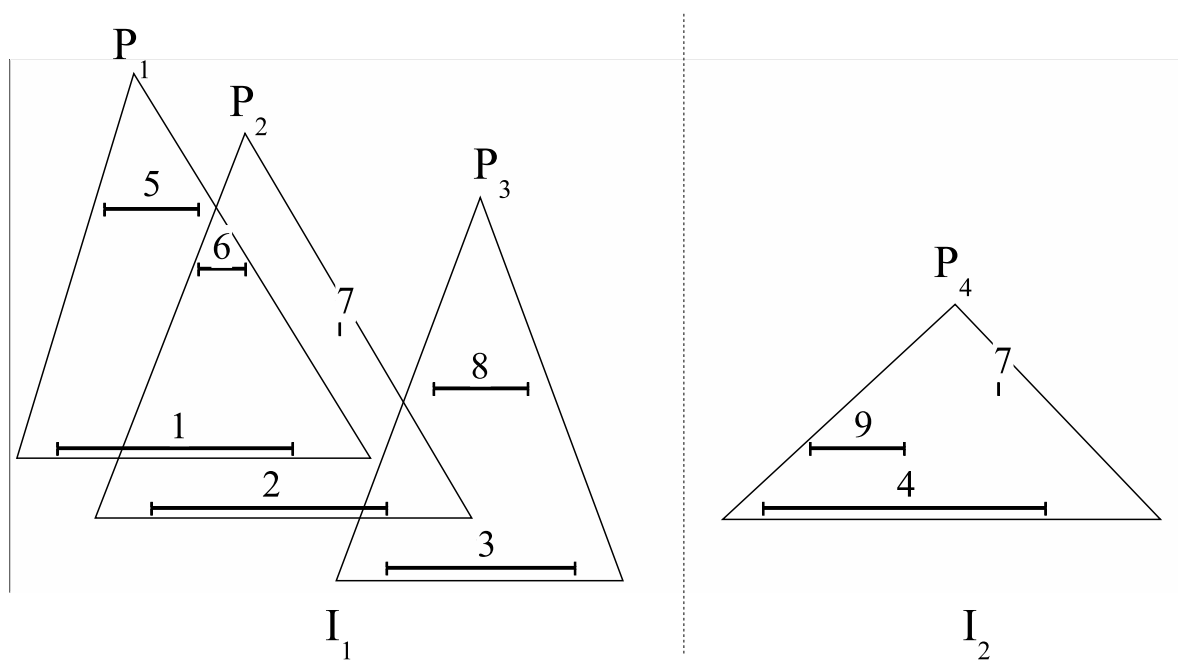

Fig. 5. Interval structures $I_{1}$ and $I_{2}$ for the problem of the figure 4

Before giving our general result, we need to consider two particular interval structures: we focus first on the case where a $F 2 \mid$ prmu $\mid C_{\max }$ problem is characterised by one single b-pyramid, then we consider the bi-pyramidal case.

\section{The mono-pyramidal case}

Let us consider a $F 2|p r m u| C_{\text {max }}$ problem with only one single base $b$ and one single b-pyramid $P_{b}$. Two cases will be distinguished either $P_{b}$ is a b-pyramid of $I_{1}$ or $P_{b}$ is a b-pyramid of $I_{2}$.

\section{1 $P_{b}$ is a b-pyramid of $I_{1}$}

In this case, the following theorem can be stated.

Theorem 1. Any job sequence which places the base $b$ in the first position and the other jobs in any order is optimal with:

$$
C_{\max }=p_{b 1}+\sum_{j \in J} p_{j 2}
$$


Proof. We must prove that there is necessarily no idle time on $M_{2}$ when the base $b$ is sequenced first. First, as $b$ is the only base, any job $j \in J-\{b\}$ of $I_{1}$ obviously respects the inequality $p_{b 1}<p_{j 1} \leq p_{j 2}<p_{b 2}$. As illustrated on the figure 6 , we note $\sigma$ the sub-sequence of $n$ jobs being already sequenced after the base $b$, and $\Delta t_{n}$ the idle time on $M_{1}$ (i.e. $\Delta t_{n}=C_{\sigma+\{b\}}-\sum_{j \in \sigma+\{b\}} p_{j 1}$ ). Proving that there is no idle time on $M 2$ is equivalent to prove that $\Delta t_{n} \geq p_{j 1} \forall n$ and $\forall j \in J-\sigma-\{b\}$. The case where only the base $b$ is sequenced (i.e. $\sigma=\emptyset$ ) is obvious since $\Delta t_{1}=p_{b 2}$ and therefore, $\Delta t_{1}>p_{j 1} \forall j \in J-\{b\}$. If we consider now the general case, then it is easy to see that $\Delta t_{n}=\Delta t_{n-1}+p_{j 2}-p_{j 1}$. Since $p_{j 2}-p_{j 1} \geq 0$ by hypothesis, we can deduce that $\Delta t_{n} \geq \Delta t_{n-1} \geq \cdots \geq \Delta t_{1}>$ $p_{j 1}$. Hence there is no idle time on $M_{2}$ and $C_{\max }=p_{b 1}+\sum_{j \in J} p_{j 2}$.

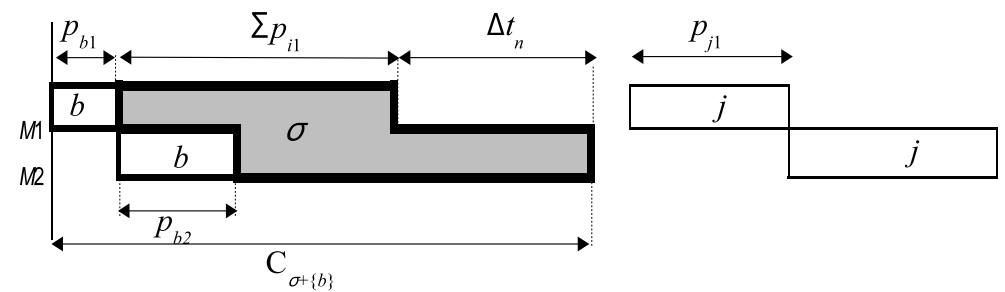

Fig. 6. Optimal job sequences for the mono-pyramidal case ( $P_{b}$ belongs to $\left.I_{1}\right)$

\section{2 $\quad P_{b}$ is a b-pyramid of $I_{2}$}

In the case where $b$ is a base of $I_{2}$, a dual theorem can be stated.

Theorem 2. Any job sequence which places the base $b$ in the last position and the other jobs in any order is optimal with :

$$
C_{\max }=p_{b 2}+\sum_{j \in J} p_{j 1}
$$

Proof. The proof is similar to the previous one by permuting the processing times $p_{j 1}$ and $p_{j 2}$ (reverse problem formulation). Therefore, the optimal job sequences are the same but in the reverse order.

\section{The bi-pyramidal case}

We assume now that the problem is characterized by two bases $b_{1}$ and $b_{2}$. We consider two cases either $b_{1}$ and $b_{2}$ belongs to the same interval structure $\left(I_{1}\right.$ or $I_{2}$ ) or not. 
We assume that $b_{1}$ and $b_{2}$ are two bases belonging to $I_{1}$ such that $p_{b_{1} 1} \leq p_{b_{2} 1}$. As illustrated on the figure 7 , we focus on job sequences in the form $b_{1} \prec \sigma_{1} \prec$ $b_{2} \prec \sigma_{2}$. We distinguish again two sub-cases either the relation precedes $\left(b_{1}, b_{2}\right)$ holds or not.
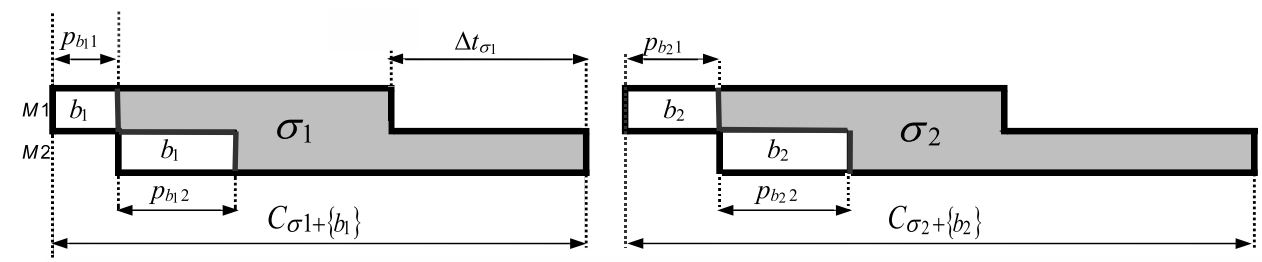

Fig. 7. Optimal job sequences for the by-pyramidal case $\left(b_{1}\right.$ and $b_{2}$ belong to $\left.I_{1}\right)$

\subsubsection{The relation precedes $\left(b_{1}, b_{2}\right)$ holds}

Since precedes $\left(b_{1}, b_{2}\right)$, obviously $P_{b_{1}} \cap P_{b_{2}}=\emptyset$ and every job $j \in J-\left\{b_{1}, b_{2}\right\}$ belongs to either $P_{b_{1}}$ or $P_{b_{2}}$. We prove the following theorem:

Theorem 3. If $P_{b_{1}}$ and $P_{b_{2}}$ are two b-pyramids of $I_{1}$ with precedes $\left(b_{1}, b_{2}\right)$, then any job sequence such that:

- $b_{1}$ precedes $b_{2}$

- all the jobs belonging to $P_{b_{1}}$ are sequenced inside $\sigma_{1}$ in any order;

- all the jobs belonging to $P_{b_{2}}$ are sequenced inside $\sigma_{2}$ in any order;

is optimal with:

$$
C_{\text {max }}=\sum_{j \in \sigma_{2}+\left\{b_{2}\right\}} p_{j 2}+\max \left(p_{b_{1} 1}+\sum_{j \in \sigma_{1}+\left\{b_{1}\right\}} p_{j 2}, \sum_{j \in \sigma_{1}+\left\{b_{1}\right\}} p_{j 1}+p_{b_{2} 1}\right) .
$$

Proof. Let us focus on the Johnson's optimal sequence which arranges jobs in the ascending order of their $p_{j 1}$. This optimal sequence is in the form $b_{1} \prec$ $\sigma_{1} \prec b_{2} \prec \sigma_{2}$ and obvioulsy respects the three conditions of the theorem 3 since $b_{1}$ precedes $b_{2}$, any job $j \in P_{b_{1}}$ is assigned to $\sigma_{1}$ and any job $j \in P_{b_{2}}$ is assigned to $\sigma_{2}$. From the theorem 1, it can be stated that the values of $C_{\sigma_{1}+\left\{b_{1}\right\}}$ and $C_{\sigma_{2}+\left\{b_{2}\right\}}$ (hence the value of the optimal makespan), do not change whathever the job orders inside $\sigma_{1}$ and $\sigma_{2}$ are. Depending on the value of $\Delta t_{\sigma_{1}}$ compared to $p_{b_{2} 1}$, the optimal makespan $C_{\max }$ has two possible expressions. If $\Delta t_{\sigma_{1}} \geq p_{b_{2} 1}$ then $C_{\max }=p_{b_{1} 1}+\sum_{j \in \sigma_{1}+\left\{b_{1}\right\}} p_{j 2}+\sum_{j \in \sigma_{2}+\left\{b_{2}\right\}} p_{j 2}$. Otherwise, $C_{\text {max }}=\sum_{j \in \sigma_{1}+\left\{b_{1}\right\}} p_{j 1}+p_{b_{2} 1}+\sum_{j \in \sigma_{2}+\left\{b_{2}\right\}} p_{j 2}$. The value of the makespan is the greater between those, hence the $C_{\max }$ expression of the theorem 3 . 


\subsubsection{The relation precedes $\left(b_{1}, b_{2}\right)$ does not hold}

We assume that $b_{1}$ and $b_{2}$ are two bases of the interval structure $I_{1}$ such that $p_{b_{1} 1} \leq p_{b_{2} 1}$. Since the relation precedes $\left(b_{1}, b_{2}\right)$ does not hold, six Allen's relations remain possible: equals $\left(b_{2}, b_{1}\right)$, starts $\left(b_{2}, b_{1}\right)$, starts $\left(b_{1}, b_{2}\right)$, ends $\left(b_{2}, b_{1}\right)$, overlaps $\left(b_{1}, b_{2}\right)$ and meets $\left(b_{1}, b_{2}\right)$. For all these cases, we notice that some jobs can belong to both $P_{b_{1}}$ and $P_{b_{2}}$.

Theorem 4. If $P_{b_{1}}$ and $P_{b_{2}}$ are two b-pyramids of $I_{1}$ such that the relation precedes $\left(b_{1}, b_{2}\right)$ does not hold, then any job sequence such that:

- $b_{1}$ precedes $b_{2}$

- all the jobs only belonging to $P_{b_{1}}$ are sequenced inside $\sigma_{1}$ in any order;

- all the jobs only belonging to $P_{b_{2}}$ are sequenced inside $\sigma_{2}$ in any order;

- any job belonging both to $P_{b_{1}}$ and $P_{b_{2}}$ is sequenced anywhere either inside $\sigma_{1}$ or $\sigma_{2}$ in any order;

is optimal with:

$$
C_{\max }=p_{b_{1} 1}+\sum_{j \in J} p_{j 2}
$$

Proof. The proof is quite similar to the previous one. Indeed, we also focus on the Johnson's optimal sequence which arranges the jobs in the ascending order of their $p_{j 1}$. This optimal sequence is in the form $b_{1} \prec \sigma_{1} \prec b_{2} \prec \sigma_{2}$ (see figure 7) and obvioulsy respects the conditions of the theorem 4 since $b_{1}$ precedes $b_{2}$, jobs belonging only to $P_{b_{1}}$ are assigned to $\sigma_{1}$ and all the other jobs (belonging either to $P_{b_{1}} \cap P_{b_{2}}$ or only to $P_{b_{2}}$ ) are assigned to $\sigma_{2}$. Since $b_{1}$ and $b_{2}$ are two bases such that precedes $\left(b_{1}, b_{2}\right)$ does not hold, the relation $p_{b_{1} 1} \leq p_{b_{2} 1} \leq p_{b_{1} 2}$ stands. Therefore, according to the proof of the theorem 1 , one can deduce that $\Delta t_{\sigma_{1}} \geq p_{b_{2} 1}$. Hence the optimal makespan value is $C_{\max }=C_{\sigma_{1}+\left\{b_{1}\right\}}+C_{\sigma_{2}+\left\{b_{2}\right\}}-p_{b_{2} 1}=p_{b_{1} 1}+\sum_{j \in \sigma_{1}+\left\{b_{1}\right\}} p_{j 2}+\sum_{j \in \sigma_{2}+\left\{b_{2}\right\}} p_{j 2}$. From the theorem 1, it can be stated that the values of $C_{\sigma_{1}+\left\{b_{1}\right\}}$ and $C_{\sigma_{2}+\left\{b_{2}\right\}}$ (hence the one of the optimal makespan), do not change whathever the job orders inside $\sigma_{1}$ and $\sigma_{2}$ are. Moreover, scheduling a job belonging both to $P_{b_{1}}$ and $P_{b_{2}}$ in $\sigma_{1}$ (instead of $\sigma_{2}$ as in the Johnson's sequence) does not decrease $\Delta t_{\sigma_{1}}$. So the relation $\Delta t_{\sigma_{1}} \geq p_{b_{2} 1}$ still holds and the $C_{\max }$ value does not change. Lastly, in the particular case where $p_{b_{1} 1}=p_{b_{2} 1}$, the base indexes can be swapped in the theorem.

\subsection{The bases $b_{1}$ and $b_{2}$ belong to the same interval structure $I_{2}$}

We assume here that $b_{1}$ and $b_{2}$ are two bases belonging to $I_{2}$ such that $p_{b_{1} 2} \geq p_{b_{2} 2}$. Then the two following theorems can be directly stated since 
their proof is similar to both previous ones, by considering the reverse formulation of the problem. In this case, the structure of the optimal sequences is $\sigma_{1} \prec b_{1} \prec \sigma_{2} \prec b_{2}$.

Theorem 5. If $P_{b_{1}}$ and $P_{b_{2}}$ are two b-pyramids of $I_{2}$ with precedes $\left(b_{2}, b_{1}\right)$, then any job sequence such that:

- $b_{1}$ precedes $b_{2}$;

- all the jobs belonging to $P_{b_{1}}$ are sequenced inside $\sigma_{1}$ in any order;

- all the jobs belonging to $P_{b_{2}}$ are sequenced inside $\sigma_{2}$ in any order;

is optimal with:

$$
C_{\text {max }}=\sum_{j \in \sigma_{1}+\left\{b_{1}\right\}} p_{j 1}+\max \left(p_{b_{2} 2}+\sum_{j \in \sigma_{2}+\left\{b_{2}\right\}} p_{j 1}, \sum_{j \in \sigma_{2}+\left\{b_{2}\right\}} p_{j 2}+p_{b_{1} 2}\right) .
$$

Theorem 6. If $P_{b_{1}}$ and $P_{b_{2}}$ are two b-pyramids of $I_{2}$ such that the relation precedes $\left(b_{2}, b_{1}\right)$ does not hold, then any job sequence such that:

- $b_{1}$ precedes $b_{2}$;

- all the jobs only belonging to $P_{b_{1}}$ are sequenced inside $\sigma_{1}$ in any order;

- all the jobs only belonging to $P_{b_{2}}$ are sequenced inside $\sigma_{2}$ in any order;

- any job belonging both to $P_{b_{1}}$ and $P_{b_{2}}$ is sequenced anywhere either inside $\sigma_{1}$ or $\sigma_{2}$ in any order;

is optimal with:

$$
C_{\max }=p_{b_{2} 2}+\sum_{j \in J} p_{j 1}
$$

5.3 The bases $b_{1}$ and $b_{2}$ do not belong to the same interval structure

In this section, we assume that $b_{1}$ is a base of $I_{1}$ and $b_{2}$ is a base of $I_{2}$. We notice that only the jobs such that $p_{j 1}=p_{j 2}$ can belong both to $P_{b_{1}}$ and $P_{b_{2}}$ (their corresponding interval is a point). As illustrated on the figure 8 , we focus on job sequences in the form $b_{1} \prec \sigma_{1} \prec \sigma_{2} \prec b_{2}$. Then we state the following theorem:

Theorem 7. If $P_{b_{1}}$ is a b-pyramid of $I_{1}$ and $P_{b_{2}}$ is a b-pyramid of $I_{2}$, then any job sequence such that:

- $b_{1}$ precedes $b_{2}$

- all the jobs only belonging to $P_{b_{1}}$ are sequenced inside $\sigma_{1}$ in any order;

- all the jobs only belonging to $P_{b_{2}}$ are sequenced inside $\sigma_{2}$ in any order;

- any job belonging both to $P_{b_{1}}$ and $P_{b_{2}}$ is sequenced anywhere either inside $\sigma_{1}$ or $\sigma_{2}$ in any order; 
is optimal with:

$$
C_{\text {max }}=p_{b_{1} 1}+p_{b_{2} 2}+\max \left(\sum_{j \in \sigma_{1} \cup \sigma_{2}} p_{j 1}+p_{b_{2} 1}, \sum_{j \in \sigma_{1} \cup \sigma_{2}} p_{j 2}+p_{b_{1} 2}\right) .
$$
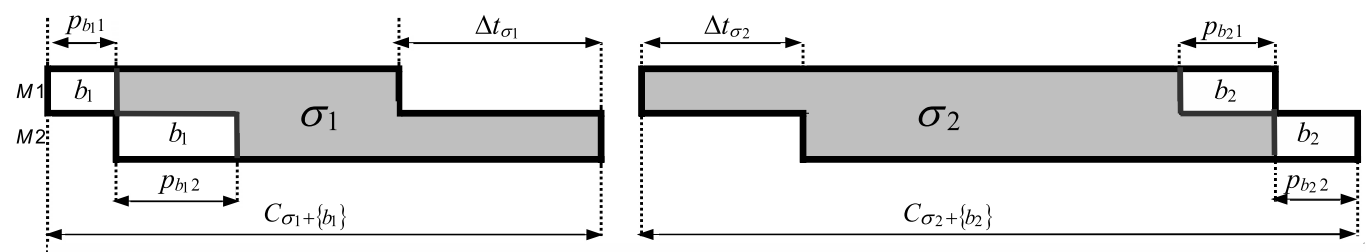

Fig. 8. Optimal job sequences for the by-pyramidal case $\left(b_{1}\right.$ belong to $I_{1}$ and $b_{2}$ belong to $I_{2}$ )

Proof. Once again, let us consider the Johnson's optimal sequence which arranges the jobs belonging to $I_{1}$ in the ascending order of their $p_{j 1}$ and the jobs belonging to $I_{2}$ in the descending order of their $p_{j 2}$. This optimal sequence obvioulsy respects the three conditions of the theorem 7 since $b_{1}$ precedes $b_{2}$, any job $j \in P_{b_{1}}$ is assigned to $\sigma_{1}$ and any job $j \in P_{b_{2}}$ is assigned to $\sigma_{2}$. From the theorem 1 , one can see that the values of $C_{\sigma_{1}+\left\{b_{1}\right\}}$ and $C_{\sigma_{2}+\left\{b_{2}\right\}}$ (hence the one of the optimal makespan), do not change whathever the job orders inside $\sigma_{1}$ and $\sigma_{2}$ are. Additionally, if a job $j$ belongs to both $P_{b_{1}}$ and $P_{b_{2}}$ $\left(p_{j 1}=p_{j 2}\right)$, then it can be assigned either to $\sigma_{1}$ or $\sigma_{2}$ since whatever the assignment is, it neither modifies the values of $\Delta t_{\sigma_{1}}$ and $\Delta t_{\sigma_{2}}$, nor the one of the makespan. Depending on the value of $\Delta t_{\sigma_{1}}$ compared to $\Delta t_{\sigma_{2}}$, two cases may happen. If $\Delta t_{\sigma_{1}} \geq \Delta t_{\sigma_{2}}$ then $C_{\max }=p_{b_{1} 1}+p_{b_{1} 2}+\sum_{j \in \sigma_{1} \cup \sigma_{2}} p_{j 2}+p_{b_{2} 2}$. Otherwise, $C_{\max }=p_{b_{1} 1}+\sum_{j \in \sigma_{1} \cup \sigma_{2}} p_{j 1}+p_{b_{2} 1}+p_{b_{2} 2}$. Hence the general $C_{\max }$ expression of the theorem 7 .

\section{A sufficient condition of optimality for $F 2|p r m u| C_{\max }$}

Now we consider the general case where each interval structure $I_{1}$ and $I_{2}$ is characterized by any number of b-pyramids. As illustrated on the figure 9, we denote $\sigma_{j}$, with $j \in\left[1, n_{1}\right]$, the sub-sequence of jobs located between the bases $b_{j}$ and $b_{j+1}$, provided that the bases of $I_{1}$ have been indexed according to the ascending order of their $p_{j 1}$ (in any order in case of equality). Similarly, we denote $\sigma_{k}$, with $k \in\left[n_{1}+1, n_{1}+n_{2}\right]$, the sub-sequence of jobs located between the bases $b_{k-1}$ and $b_{k}$, provided that the bases of $I_{2}$ have been indexed according to the descending order of their $p_{j 2}$ (in any order in case of equality). We focus on job sequences in the form: $b_{1} \prec \sigma_{1} \prec b_{2} \prec \sigma_{2} \prec \cdots \prec b_{n_{1}} \prec \sigma_{n_{1}} \prec$ $\sigma_{n_{1}+1} \prec b_{n_{1}+1} \prec \cdots \prec \sigma_{n_{1}+n_{2}} \prec b_{n_{1}+n_{2}}$. 


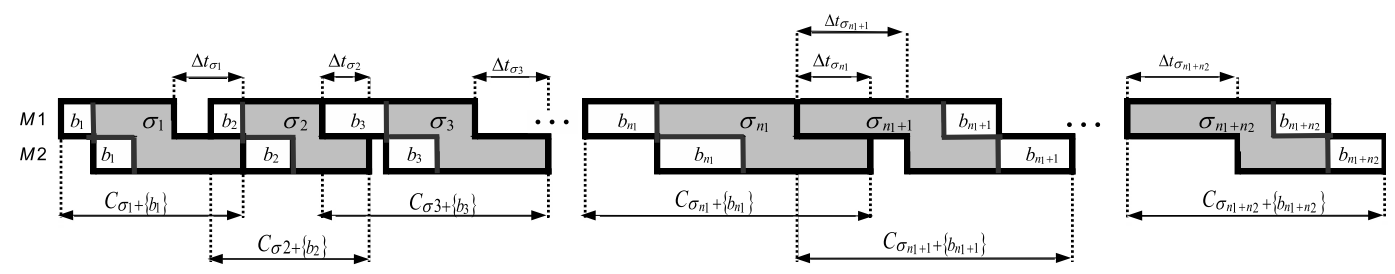

Fig. 9. Optimal job sequences for the general case

For each job $j$, we denote $u(j)$ (respectively $v(j)$ ) the index of the base of the first (respectively the last) b-pyramid to which the job $j$ belongs. For a base $b_{i}$, we set $u\left(b_{i}\right)=v\left(b_{i}\right)=i$. Then we state the following general theorem.

Theorem 8. Any job sequence such that:

- the bases of $I_{1}$ and $I_{2}$ are sequenced according to the ascending order of their indexes;

- any job $j$ is sequenced inside any sub-sequence from $\sigma_{u(j)}$ to $\sigma_{v(j)}$ in any order;

is optimal with:

$$
\begin{aligned}
C_{\text {max }}= & C_{\sigma_{1}+\left\{b_{1}\right\}}+ \\
& \max \left(C_{\sigma_{2}+\left\{b_{2}\right\}}-p_{b_{2} 1}, C_{\sigma_{2}+\left\{b_{2}\right\}}-\Delta t_{\sigma_{1}}\right)+ \\
& \max \left(C_{\sigma_{3}+\left\{b_{3}\right\}}-p_{b_{3} 1}, C_{\sigma_{3}+\left\{b_{3}\right\}}-\Delta t_{\sigma_{2}}\right)+ \\
& \cdots+ \\
& \max \left(C_{\sigma_{n_{1}}+\left\{b_{n_{1}}\right\}}-p_{b_{n_{1}} 1}, C_{\sigma_{n_{1}}+\left\{b_{n_{1}}\right\}}-\Delta t_{\sigma_{n_{1}-1}}\right)+ \\
& \max \left(C_{\sigma_{n_{1}+1}+\left\{b_{n_{1}+1}\right\}}-\Delta t_{\sigma_{n_{1}+1}}, C_{\sigma_{n_{1}+1}+\left\{b_{n_{1}+1}\right\}}-\Delta t_{\sigma_{n_{1}}}\right)+ \\
& \max \left(C_{\sigma_{n_{1}+2}+\left\{b_{n_{1}+2}\right\}}-\Delta t_{\sigma_{n_{1}+2}}, C_{\sigma_{n_{1}+2}+\left\{b_{n_{1}+2}\right\}}-p_{b_{n_{1}+1} 2}\right)+ \\
& \cdots+ \\
& \max \left(C_{\sigma_{n_{1}+n_{2}-1}+\left\{b_{n_{1}+n_{2}-1}\right\}}-\Delta t_{\sigma_{n_{1}+n_{2}-1}}, C_{\sigma_{n_{1}+n_{2}-1}+\left\{b_{n_{1}+n_{2}-1}\right\}}-p_{b_{n_{1}+n_{2}-2} 2}\right)+ \\
& \max \left(C_{\sigma_{n_{1}+n_{2}}+\left\{b_{n_{1}+n_{2}}\right\}}-\Delta t_{\sigma_{n_{1}+n_{2}}}, C_{\sigma_{n_{1}+n_{2}}+\left\{b_{n_{1}+n_{2}}\right\}}-p_{b_{n_{1}+n_{2}-1} 2}\right)
\end{aligned}
$$

with:

$$
\begin{gathered}
C_{\sigma_{i}+\left\{b_{i}\right\}}= \begin{cases}p_{b_{i} 1}+\sum_{j \in \sigma_{i}+\left\{b_{i}\right\}} p_{j 2} & \text { if } i \in\left[1, n_{1}\right] \\
p_{b_{i} 2}+\sum_{j \in \sigma_{i}+\left\{b_{i}\right\}} p_{j 1} \text { if } i \in\left[n_{1}+1, n_{1}+n_{2}\right]\end{cases} \\
\Delta t_{\sigma_{i}}= \begin{cases}C_{\sigma_{i}+\left\{b_{i}\right\}}-\sum_{j \in \sigma_{i}+\left\{b_{i}\right\}} p_{j 1} \text { if } i \in\left[1, n_{1}\right] \\
C_{\sigma_{i}+\left\{b_{i}\right\}}-\sum_{j \in \sigma_{i}+\left\{b_{i}\right\}} p_{j 2} \text { if } i \in\left[n_{1}+1, n_{1}+n_{2}\right]\end{cases}
\end{gathered}
$$

Proof. We focus on the Johnson's sequence which arranges first the jobs of $I_{1}$, according to the ascending order of their $p_{j 1}$, then the jobs of $I_{2}$, according to 
the descending order of their $p_{j 2}$. This optimal sequence respects the theorem 8 since the base order matches the increasing order of the base index, any job $j \in I_{1}$ is assigned to $\sigma_{v(j)}$ and any job $j \in I_{2}$ is assigned to $\sigma_{u(j)}$. From the theorems $3,4,5,6$ and 7 , one can easily deduce the optimal makespan expression given in the theorem 8. Moreover, since this expression gives the optimal makespan value for the particular previous Johnson's sequence, it also can be deduced that this value does not changed for any assignement of a non-base job $j$ ranging from $\sigma_{u(j)}$ to $\sigma_{v(j)}$ (as seen in the proof of the theorem 4).

Now we intend to prove that the partial order defined by the theorem 8 is an extension of the partial order defined by the Johnson's rule.

Corollary 1. Any Johnson's sequence is necessarily included in the set of job sequences characterized by the theorem 8.

Proof. From the theorem 8, we state that a job $i$ cannot precede a job $j$ in any optimal sequence if and only if $u(i)>v(j)$. Indeed, if $u(i) \leq v(j)$ then either $u(i) \leq v(i)<u(j) \leq v(j)$ and $i$ precedes $j$ in every job sequence, or $u(i) \leq u(j) \leq v(i) \leq v(j)$ and job sequences exists such that $i$ and $j$ are assigned to the same sub-sequence $\sigma_{k}$ with $u(j) \leq k \leq v(i)$ (hence $i$ and $j$ can be sequenced in any order). The relation $u(i)>v(j)$ (i.e. $i \nprec j$ ) only stands in one of the following cases:

- $j$ belongs to $I_{1}$ (but not to $I_{2}$ ) and $i$ belongs to $I_{2}$ (but not to $I_{1}$ ), hence $p_{j 2}>p_{j 1}$ and $p_{i 1}>p_{i 2}$

- $i$ and $j$ both belong to $I_{1}$ and either $p_{j 1}<p_{i 1} \leq p_{j 2}<p_{i 2}$ (i.e. overlaps $(j, i)$ or meets $(j, i))$ or $p_{j 1}<p_{j 2}<p_{i 1}<p_{i 2}$ (i.e. precedes $\left.(j, i)\right)$, and there does not exist any base $b$ such that both during $(i, b)$ and during $(j, b)$ ( $i$ and $j$ do not belong to any common b-pyramid);

- $i$ and $j$ both belong to $I_{2}$ and either $p_{i 2}<p_{j 2} \leq p_{i 1}<p_{j 1}$ (i.e.overlaps $(i, j)$ or meets $(i, j))$ or $p_{i 2}<p_{i 1}<p_{j 2}<p_{j 1}$ (i.e. $\operatorname{precedes}(i, j)$ ), and there does not exist any base $b$ such that both during $(i, b)$ and during $(j, b)$ ( $i$ and $j$ do not belong to any common b-pyramid);

Thus one can see that in any above case the inequality $\min \left(p_{i 1}, p_{j 2}\right)>\min \left(p_{i 2}, p_{j 1}\right)$ always holds. Therefore the theorem 8 only discards job precedences which do not satisfy the Johnson's rule. Hence the Johnson's sequences are conserved.

Also interesting is the impressive number of job sequences characterized by our theorem. Let $S$ be the set of all the possible job assignments inside the sub-sequences $\sigma$. The cardinality of $S$ is $\prod_{q=1}^{m} q^{n_{q}}$ [14], where $m$ is the number of b-pyramids and $n_{q}$, the number of jobs belonging to exactly $q$ b-pyramids. 
For a particular job assignment $s \in S$, let $n_{i}(s)$ be the total number of jobs assigned to $\sigma_{i}$ with respect of the theorem. Then the total number of job permutations is $\prod_{i=1}^{m}\left(n_{i}(s) !\right)$. Hence, the total number of characterized sequences is $\sum_{s \in S}\left(\prod_{i=1}^{m} n_{i}(s) !\right)$.

As illustration, for a problem with 20 jobs such that $m=4, u(j)=v(j) \forall j \in J$ and $n_{i}=4$, the theorem characterizes $(4 !)^{4}=331776$ optimal sequences. Now for the same problem, if we assume that one (and only one) job belongs to two b-pyramids, then the number of characterizd optimal sequences becomes $(4 !)^{4}+(3 ! * 5 ! * 4 ! * 4 !)=746496$.

\section{Example}

For theorem 8 illustration, let us take again the example of the figure 4 . The value of the functions $u(j)$ and $v(j)$ are given for each job $j$ on the table of the figure 10 .

\begin{tabular}{l|l|l|l|l|l|l|l|l|l} 
Jobs $j$ & 1 & 2 & 3 & 4 & 5 & 6 & 7 & 8 & 9 \\
\hline$u(j)$ & 1 & 2 & 3 & 4 & 1 & 1 & 2 & 3 & 4 \\
\hline$v(j)$ & 1 & 2 & 3 & 4 & 1 & 2 & 4 & 3 & 4
\end{tabular}

Fig. 10. $u(j)$ and $v(j)$ values

According to the theorem, we focus on job sequences in the form $b_{1} \prec \sigma_{1} \prec$ $b_{2} \prec \sigma_{2} \prec b_{3} \prec \sigma_{3} \prec \sigma_{4} \prec b_{4}$. Regarding the table of the figure 10, the possible assignments of the jobs are $5 \in \sigma_{1}, 6 \in \sigma_{1}$ or $6 \in \sigma_{2}, 7 \in \sigma_{2}$ or $7 \in \sigma_{3}$ or $7 \in \sigma_{4}, 8 \in \sigma_{3}$ and $9 \in \sigma_{4}$. We recall that the order of jobs inside each sub-sequence $\sigma_{i}$ does not impact the makespan value. Therefore, enumerating all the possibilities, we find thirteen optimal job sequences with the optimal makespan $C_{\max }=49: \quad 1 \prec 5 \prec 6 \prec 2 \prec 7 \prec 3 \prec 8 \prec 9 \prec 4$, $1 \prec 6 \prec 5 \prec 2 \prec 7 \prec 3 \prec 8 \prec 9 \prec 4, \quad 1 \prec 5 \prec 6 \prec 2 \prec 3 \prec 8 \prec 7 \prec 9 \prec 4$, $1 \prec 6 \prec 5 \prec 2 \prec 3 \prec 8 \prec 7 \prec 9 \prec 4, \quad 1 \prec 5 \prec 6 \prec 2 \prec 3 \prec 7 \prec 8 \prec 9 \prec 4$, $1 \prec 6 \prec 5 \prec 2 \prec 3 \prec 7 \prec 8 \prec 9 \prec 4, \quad 1 \prec 5 \prec 6 \prec 2 \prec 3 \prec 8 \prec 9 \prec 7 \prec 4$, $1 \prec 6 \prec 5 \prec 2 \prec 3 \prec 8 \prec 9 \prec 7 \prec 4, \quad 1 \prec 5 \prec 2 \prec 6 \prec 7 \prec 3 \prec 8 \prec 9 \prec 4^{*}$, $1 \prec 5 \prec 2 \prec 7 \prec 6 \prec 3 \prec 8 \prec 9 \prec 4, \quad 1 \prec 5 \prec 2 \prec 6 \prec 3 \prec 7 \prec 8 \prec 9 \prec 4^{*}$, $1 \prec 5 \prec 2 \prec 6 \prec 3 \prec 8 \prec 7 \prec 9 \prec 4^{*}, \quad 1 \prec 5 \prec 2 \prec 6 \prec 3 \prec 8 \prec 9 \prec 7 \prec 4$. For this problem instance, only the three asterisked sequences satisfy the Johnson's rule.

In order to illustrate the robustness of our approach, we slightly increase some job processing times in the above example. Then we analyse the consequences on the above set of optimal job sequences. 
First let us suppose that, due to one disruption, $p_{51}$ increases so that $p_{51}=3$ (instead of 2). This modification does not affect the interval structures $I_{1}$ and $I_{2}$ since the Allen's relations between any pair of job intervals are maintained. Therefore the above set of optimal sequences is kept unchanged, as well as the $C_{\max }$ value (since $C_{\max }$ does not depend on $p_{51}$ for this problem instance).

Now if we set $p_{52}=5$ (instead of 4$)$ then the interval structures $I_{1}$ and $I_{2}$ are again unchanged, as well as the set of optimal sequences. Nevertheless, the optimal makespan value is now $C_{\max }=60$ (instead of 59) for any job sequence.

Now we suppose that $p_{62}$ increases so that $p_{62}=7$ (instead of 5$)$. In this case, the interval structure $I_{1}$ is modified because the job 6 does not belong any more to the b-pyramid $P_{b_{1}}$. Therefore any job sequence which assigns the job 6 to $\sigma_{1}$ has to be removed from the set of optimal sequences. Thus it only remains five optimal job sequences with the optimal makespan $C_{\max }=62$ :

$1 \prec 5 \prec 2 \prec 6 \prec 7 \prec 3 \prec 8 \prec 9 \prec 4, \quad 1 \prec 5 \prec 2 \prec 7 \prec 6 \prec 3 \prec 8 \prec 9 \prec 4$, $1 \prec 5 \prec 2 \prec 6 \prec 3 \prec 7 \prec 8 \prec 9 \prec 4, \quad 1 \prec 5 \prec 2 \prec 6 \prec 3 \prec 8 \prec 7 \prec 9 \prec 4$, $1 \prec 5 \prec 2 \prec 6 \prec 3 \prec 8 \prec 9 \prec 7 \prec 4$.

We see through this simple scenario that our approach is relatively insensitive to data variations as long as these variations do not modify the bases of the interval structures. Of course if this condition is not respected then a new interval analysis has to be performed in order to re-compute dynamically the new b-pyramids.

\section{Conclusion}

In this paper, we established a new sufficient condition of optimality for the $F 2 \mid$ prmu $\mid C_{\text {max }}$ problem. Based on two interval structures associated to the problem, the sufficient condition of optimality characterizes a set of optimal job sequences by determining the bases and the related b-pyramids of the interval structures. This sufficient condition is quite efficient since it ensures that any Johnson's sequence is always included in the set of characterized sequences, together with numerous other optimal job sequences. Furthermore, as the set of optimal sequences remains unchanged as long as the relative order of the processing times is conserved, this approach is relatively insensitive to processing time variations. For the future works, we want to reuse the results of this paper for solving more complex problems, starting with the $F 2\left|r_{i}\right| C_{\max }$ one, in order to define some new sufficient or dominant partial orders. 


\section{References}

[1] Aloulou, M. A., Portmann, M.-C., \& Vignier, A., Predictive-reactive scheduling for the single machine problem, 2002, $8^{\text {th }}$ Workshop on Project Management and Scheduling, Valencia, 3-5 april, 39-42.

[2] Allen, J., An interval based representation of temporal knowledge, 1981, Proceedings of the $7^{\text {th }}$ IJCAI, Vancouver, Canada, 221-226.

[3] Artigues, C., \& Roubellat, F., Characterization of a set of schedules in resourceconstrained multi-project scheduling problem, 1999, International Journal of Industrial Engineering - Theory, Applications and Practice, 6(2), 112-122.

[4] Baptiste, P., Sub-optimal groups of sequences in the classical flow-shop F2 $\| C_{\max }, 1996,5^{\text {th }}$ Workshop on Project Management and Scheduling, Poznan, 11-13 april, 27-30.

[5] Belman, R., Esogbue, A.O, \& Nabeshima, I., Mathematical aspects of scheduling and applications, 1982, Pergamon press, Oxford.

[6] Billaut, J.-C, \& Lopez, P., Enumeration of all optimal sequences in the twomachine flowshop, 1998, Proceedings CESA'98 Computational Engineering in Systems Applications - Symposium on Industrial and Manufacturing Systems, Nabeul-Hammamet (Tunisia), 378-382.

[7] Billaut, J.-C, \& Roubellat, F., Characterization of a set of schedules in a multiple resource context, 1996, Journal of Decision Systems, 5(1-2), 95-109.

[8] Benoit, M., \& Billaut, J.-C, Characterization of the optimal solutions of the $F 2 \| C_{\max }$ scheduling problem, 2000, Second Conference on Management and Control of Production and Logistics (MCPL'2000), IFAC / IFIP / IEEE, Grenoble, proceedings on CD-ROM.

[9] Briand, C., La, H.T., \& Erschler, J., Une approche pour l'ordonnancement robuste de tâches sur une machine, 2003, Proceedings MOSIM'03, Toulouse 2003, 205-211 (in french).

[10] Briand, C., Despontin, E., \& Roubellat, F., Scheduling with time lags and preferences : a heuristic, 2002, $8^{\text {th }}$ Workshop on Project Management and Scheduling, Valencia, 3-5 april, 77-80.

[11] Cheng, J., Steiner, G., \& Stephenson, P., Fast algorithms to minimize the makespan or maximum lateness in the two-machine flow shop with release times, 2002, Journal of Scheduling, 5:71-92.

[12] Conway, R.W., Maxwel, W.L., \& Miller, L. W., Theory of scheduling, 1967, Addison-Wesley, Reading, Massachusetts.

[13] Davenport, A.J., \& Beck, J.C., A survey of techniques for scheduling with uncertainty, 2000, unpublished, available at http://www.eil.utoronto.ca/profiles/chris/chris.papers.html. 
[14] Erschler, J., Fontan, G., Merce, C., \& Roubellat, F., A new dominance concept in scheduling $\mathrm{n}$ jobs on a single machine with ready times and due dates, 1883, Operations Research, 1, 114-127.

[15] Esquirol, P., Lopez, P., \& Mancel, P., Représentation et traitement du temps en ordonnancement, 1999, Rapport LAAS N99455 (in french).

[16] Esswein, C., \& Billaut, J.-C, Trade-off between flexibility and maximum completion time in the two-machine flowshop scheduling problem, 2002, International Symposium on Combinatorial Optimisation, Paris (France).

[17] Herroelen, W., \& Leus, R., Project scheduling under uncertainty - survey and research potentials, 2003, European Journal of Operational Resarch, to appear.

[18] Herroelen, W., \& Leus, R., Robust and reactive project scheduling - a review and classification of procedures, 2004, International Journal of Production Research, vol. 42, 8, 1599-1620.

[19] Johnson, S.M., Optimal two and three stage production schedules with set-up times included, 1954, Naval research Logistics Quaterly, 1, 61-68.

[20] Le Gall, A., Un système interactif d'aide à la décision pour l'ordonnancement et le pilotage en temps réel d'ateliers, 1989, PhD Thesis, Université Paul Sabatier, Toulouse, France (in french).

[21] Mauguière, P., Billaut, J.-C., \& Artigues, C., Grouping on a single machine with heads and tails to represent a family of dominant schedules, 2002, $8^{\text {th }}$ Workshop on Project Management and Scheduling, Valencia, 3-5 april, 265-269..

[22] Thomas, V., Aide à la décision pour l'ordonnancement d'ateliers en temps réel, 1980, PhD Thesis, Université Paul Sabatier, Toulouse, France (in french).

[23] Wu, D., Byeon, E.S., \& Storer, R.H., A graph-theoretic decomposition of the job shop scheduling problem to achieve scheduling robustness, 1999, Operations Research, 1, 113-124. 